\title{
MedienPädagogik
}

Zeitschrift für Theorie und Praxis der Medienbildung

Themenheft Nr. 1: Medienkompetenz

Herausgegeben von Lothar Mikos

\section{Editorial: Medienkompetenz}

Heinz Moser

Für die deutschsprachige Medienpädagogik beginnt das Jahr 2000 mit der ersten wissenschaftlichen Online-Zeitschrift, die den theoretischen Diskurs über Medien und Medienpädagogik in den Mittelpunkt ihres Programms stellt. Sie wird herausgegeben von der AG Medienpädagogik der Deutschen Gesellschaft für Erziehungswissenschaft und dem Fachbereich Medienbildung des Pestalozzianums Zürich. Damit erhält unsere Disziplin ein Publikationsorgan, das es ihr ermöglicht, einen Diskurs besser zu vernetzen und öffentlich zu machen, der sich im letzten Jahrhundert erst ganz allmählich entwickelt hat.

Am Anfang des 20. Jahrhunderts waren es vor allem die industrialisierten und für die Bedürfnisse eines Massenmarktes konfektionierten Druckerzeugnisse, welche die Agenda der Medienpädagogik bestimmten. Man diskutierte die Problematik einer massenhaft verbreiteten «Schundliteratur» und forderte eine «unverderbte» Kinder- und Jugendliteratur. Andere Positionen - wie diejenige von Heinrich Wolgast (1910) - lehnten hingegen eine vorab moralisch inspirierte spezifische Kinder- und Jugendliteratur ab und zweifelten an ihrem ästhetischen Wert. In den zwanziger Jahren kam der Aspekt des Films hinzu - ebenfalls vorwiegend unter dem Gesichtspunkt des Kinder- und Jugendschutzes (Popert 1927). Medienkompetenz war indessen bei all diesen Diskussionen noch kaum ein Thema; vielmehr betrachtete man die Kinder und Jugendlichen als wehrlose Opfer von Erzeugnissen, die allein dem Kommerz verpflichtet waren.

Generell war die Medienpädagogik sehr lange einer normativen Pädagogik verpflichtet, die glaubte, man müsse Kinder und Jugendliche vorwiegend vor einer Reizüberflutung durch - moralisch verwerfliche - Medien, den so genannten «geheimen Miterziehern» (Beer 1960), schützen. Dementsprechend war der theoretische Gehalt des Diskurses über Medien relativ schwach ausgeprägt. Die Überwindung dieser normativen Positionen stand insbesondere mit einer verstärkten empirischen Ausrichtung der Medienpädagogik im Zusammenhang, wie sie im Rahmen der «realistischen Wende» der Erziehungswissenschaft ab den Sechzigerjahren verstärkt auf den Plan trat. Dabei waren auch diese Bestrebungen zu Beginn noch stark auf die bewahrpädagogische Grundstruktur bezogen, wie sie mit den Konzepten der damaligen Wirkungsforschung verbunden war. Man wollte die problematischen Wirkungen der Medien - und nun vor allem des Fernsehens - empirisch erhärten; doch dies war nicht möglich, ohne gehaltvolle empirische Hypothesen zu bilden. Um nur ein prominentes Beispiel zu nennen: Die Untersuchungen von Herta Sturm (Sturm 1985) zur «fehlenden Halbsekunde» konnten nicht einfach normative Empfehlungen formulieren, sondern mussten das Konstrukt der Medienwirkung auf empirisch gehaltvolle Weise

Moser, Heinz. 2000. «Editorial: Medienkompetenz». MedienPädagogik 1, (März), i-iii. https://doi.org/10.21240/mpaed/01/2000.03.24.X. 
ausdifferenzieren. Aufgrund der damit gesetzten Ansprüche waren einfache Antworten immer weniger möglich.

So betonte Dieter Baacke 1973, der Begriff der «Massenmedien» sei kein kulturkritischer, sondern ein deskriptiver Begriff. Es sei vielmehr problematisch, Begriffe wie «Massengesellschaft» zu assoziieren, da Öffentlichkeit in der heutigen Gesellschaft keine ungegliederte und amorphe Masse darstelle (vgl. Baacke 1973, S. 13 f.). Immer stärker fanden seither Diskussionen aus dem medienwissenschaftlichen und kulturellen Bereich Eingang in die medienpädagogische Disziplin: So waren Theoreme mit wie die Wissenskluft-Theorie oder das im Rahmen der cultural studies entwickelte Konzept des active reader geeignet, die Annahme gleichförmiger Wirkungen von Medien auf die Population der Kinder und Jugendlichen zu hinterfragen und die Position des Rezipienten nicht einfach aus einer Opferolle heraus zu thematisieren.

In den letzten zwanzig Jahren hat zudem das neue Medium des Computers pädagogische Fragen aufgeworfen, die auf verschiedensten Ebenen Diskussionen auslösten. Gesellschaftlich stand zur Diskussion, inwieweit die heutige Gesellschaft als Medien- oder Informationsgesellschaft bezeichnet werden kann, und wie Kinder und Jugendliche darauf vorzubereiten sind. Auf didaktischer Ebene ging es um die Frage, wie die neuen Medien in die Schule zu integrieren sind, bzw. wie sich das Lernen durch diese verändert.

Alle diese Themen und Diskussionen finden gegenwärtig Ausdruck in vielfältigen Publikationen und Beiträgen in Fachzeitschriften. Doch es fehlt ein Gefäss, welches schwergewichtig und systematisch den Diskurs der Medienpädagogik dokumentiert und fördert. Diese Lücke soll die Zeitschrift MedienPädagogik füllen, deren erste Ausgabe Sie hier auf dem Bildschirm vorliegen haben. Bewusst haben sich die beteiligten Partner für eine Online-Zeitschrift als Format entschieden. Das Gewicht der elektronischen Medien in der medienpädagogischen Diskussion kommt dadurch sozusagen sinnlich zum Ausdruck.

Inhaltlich nimmt die vorliegende erste Ausgabe ein Kernthema der heutigen Diskussion um die Medien auf, nämlich den Schlüsselbegriff der Medienkompetenz. Die Themenwahl dieser ersten Nummer ist nicht zuletzt als Hommage an Dieter Baacke gedacht, an den leider viel zu früh verstorbenen Doyen und Mitstreiter für die Sache der Medienpädagogik, auf welchen seit den Siebzigerjahren des letzten Jahrhunderts wesentliche Impulse für die medienpädagogische Diskussion und Praxis zurückgehen.

In den Beiträgen dieser Nummer wird der von ihm geprägte Begriff der Medienkompetenz - von Kübler (1996) ironisch als «Lieblingsmetapher der Medienpädagogik» bezeichnet - thematisiert und in seiner Funktion für die aktuelle medienpädagogische Debatte deutlich gemacht. Insbesondere geht es um die Frage, inwieweit Medienkompetenzen von Kindern und Jugendlichen über Sozialisationsprozesse quasi automatisch erworben werden, bzw. welchen Stellenwert das «Erlernen» 
solcher Kompetenzen hat. Dabei wird deutlich, dass es sich nicht einfach um einen Reflexionsbegriff handelt, indem Kinder und Jugendliche über den Sinn der Medien aufgeklärt werden; vielmehr geht es immer auch um die gestalterischen und kreativen Aspekte - also um kompetentes Handeln. Diese Dimension wird gegenwärtig besonders deutlich durch die Interaktivität von Medien wie dem Internet, das Kindern und Jugendlichen eine Vielzahl von Beteiligungschancen bietet - von der Teilnahme an Chats und Online-Diskussionen bis zur eigenen Homepage.

Medienkompetenz soll aber in unserer Zeitschrift auch als inhaltlicher Anspruch verstanden werden, nämlich im Sinne eines Publikationsorgans, welches kompetent über Medien und medienpädagogische Fragen informiert sowie die wissenschaftliche Debatte weiterbringt. Auch wir setzen in diesem Zusammenhang auf die Interaktivität der Medien. In diesem Sinne freuen wir uns auf Reaktionen zu den Beiträgen dieser Nummer. Dazu ist ein Diskussionsforum eingerichtet, auf welchen die Debatte über die vorliegende Beiträge aufgenommen werden kann. In einer Online-Zeitschrift ist es möglich, solche Diskurse als integrierte Bestandteile einer Zeitschriftenausgabe zu konzipieren. Herausgeberschaft und Redaktion würden sich freuen, wenn diese Möglichkeiten von den Leserinnen und Lesern der Zeitschrift rege genutzt würden.

\section{Literatur}

Baacke, Dieter. Kommunikation und Kompetenz. München 1973.

Beer, Ulrich. Geheime Miterziehr der Jugend. Düsseldorf 1960.

Kübler, Hans-Dieter. «Kompetenz der Kompetenz der Kompetenz ... Anmerkungen zur Lieblingsmetapher der Medienpädagogik.» medien praktisch 2 (1996): $11 \mathrm{ff}$.

Popert, Hermann M. Hamburg und der Schundkampf. Zweites Buch: Filmfragen. Hamburg 1927. Wolgast, Heinrich. Das Elend unserer Jugendliteratur. Hamburg 1910. 